\title{
PREDISPOSICIÓN ADQUIRIDA AL DAÑO RENAL ASOCIADA AL CONSUMO DE TABACO
}

\section{Acquired Predisposition to Renal Damage Associated to Tobacco Consumption}

Mario DE JUAN*; Marta PRIETO; M. a Teresa HERNÁNDEZ; Francisco J. SANZ; Alfredo G. CASANOVA; Laura VICENTE; Moisés PESCADOR; Ana I. MORALES

Unidad de Toxicología del Departamento de Fisiología y Farmacología de la Universidad de Salamanca. Edificio Departamental, Lab. 223. Campus Miguel de Unamuno. 37007 Salamanca

Correo-e: mariodejuan@usal.es

RESUMEN: En los últimos años se ha visto una relación entre el tabaquismo y el daño renal. En cuanto al diagnóstico temprano del daño renal, nuestro grupo ha desarrollado el concepto de predisposición, aplicable al contexto de pacientes fumadores. Así, nuestra hipótesis es que el tabaco podría causar predisposición a sufrir daño renal agudo (DRA), lo que supondría que los fumadores podrían padecer DRA tras ser expuestos a alguna nefrotoxina, incluyendo dosis inferiores al límite tolerable. Nuestro objetivo es estudiar la relación entre los biomarcadores caracterizados en nuestro laboratorio: 1, 2 y 3 (BM1, BM2 y BM3) y el consumo de tabaco.

Se tomaron muestras de orina de pacientes fumadores y no fumadores, sin daño renal ni exposición a factores de riesgo de este. Se midieron la cotinina (ELISA), la creatininuria (kit comercial) y los biomarcadores (western blot). La creatinina plasmática se obtuvo del historial clínico de los pacientes.

Solo se vio una mayor excreción de BM3 en fumadores con respecto a no fumadores, aunque no se obtuvo una correlación con los niveles de cotinina (metabolito de nicotina que informa del grado de tabaquismo). A pesar de ello, BM3 podría suponer un buen biomarcador clínico para detectar la predisposición al DRA, lo que ayudaría a prevenir el daño renal en pacientes fumadores. 
M. DE JUAN, M. PRIETO, M. ${ }^{a}$ T. HERNÁNDEZ, F. J. SANZ, A. G. CASANOVA, L. VICENTE, M. PESCADOR, A. I. MORALES

PREDISPOSICIÓN ADQUIRIDA AL DAÑO RENAL ASOCIADA AL CONSUMO DE TABACO

Palabras clave: Predisposición adquirida; daño renal; daño renal agudo; tabaco; biomarcadores.

ABSTRACT: A relation between tobacco and renal damage has been described during the last years. Referring to early diagnosis of renal disease, our group has developed the acquired predisposition concept, which can be applied to the smoking patient context. So, our hypothesis is that tobacco may cause predisposition to acute kidney injury (AKI), which means smokers may suffer AKI after being exposed to any nephrotoxin, including undertoxicity-level doses. Our aim was to study the relationship between the predisposition biomarkers 1 (BM1), 2 (BM2) and 3 (BM3) (characterized in our laboratory) and tobacco consumption.

Urinary samples were taken from smokers and non-smokers volunteers with no renal damage nor exposure to risk factors of renal disease. The plasma creatinine of the patients was obtained from their medical history. The cotinine levels, which inform of the grade of smoking, were measured by ELISA. The urinary creatinine, used to correct urinary concentrations of biomarkers, was measured with a commercial kit based on Jaffé reaction, and the urinary biomarkers levels were measured by Western blot.

Only BM3 showed greater excretion in smoking patients than in non-smokers. However, that excretion is not related to cotinine levels. In any case, BM3 could be a good clinical biomarker of AKI predisposition which would help to prevent renal damage in smokers.

Keywords: Acquired predisposition; renal damage; acute kidney injury; tobacco smoking; biomarkers.

\section{InTRODUCCIÓN}

El tabaquismo es una enfermedad adictiva crónica responsable cada año de la muerte de más de 7 millones de personas. Las principales patologías que ocasiona son trastornos cardiovasculares, respiratorios y la aparición de tumores (OMS, 2017). La forma de consumición más habitual es el cigarrillo, que contiene unos 7000 tóxicos, de los cuales 93 son considerados por la FDA (Food and Drugs Administration) como peligrosos y potencialmente peligrosos (FDA - Cigarettes, 2017).

Sobre la relación entre daño renal y tabaquismo, aún no se conoce mucho. Pero desde que en los años 80 se observara que el tabaco hacía disminuir el filtrado glomerular en pacientes con diabetes tipo I, aumentando el riesgo de nefropatía (Christiansen, 1978), se han ido conociendo diversos mecanismos sobre 
la nefrotoxicidad del tabaco, tales como lesión glomerular, tubular o vasoconstricción renal, entre otros (Esteller et al., 1998; Repetto, 2013; FDA- Harmful and Potentially Harmful Constituents in Tobacco Products and Tobacco Smoke: Established List, 2017).

El aumento en la prevalencia mundial de la enfermedad renal crónica y su gran morbimortalidad hacen necesaria la prevención mediante el control de factores de riesgo potencialmente modificables, como el consumo de tabaco (Rocco et al., 1996). Por ello, el diagnóstico precoz del daño renal es fundamental para impedir el avance de la enfermedad. Actualmente, el marcador de daño renal más usado en clínica es la creatinina sérica $(\mathrm{CrP})$, proteína endógena de 113 Da no reabsorbible que se filtra inalterada en el glomérulo. De esta manera, midiendo la cantidad de CrP se puede establecer una relación inversa con la TFG, alterada en situaciones patológicas. El problema es que la $\mathrm{CrP}$ aumenta cuando el riñón ya ha perdido un $50 \%$ de su funcionalidad. Esto es debido a la reserva de funcionalidad renal y a la secreción tubular de creatinina, que enmascaran el descenso de la TFG (Pickering et al., 2012).

En los últimos años se han descubierto otros marcadores que se excretan en orina cuando el daño aún es temprano, como las proteínas NGAL (Neutrophil Gelatinase-Associated Lipocalin), NAG (N-Acetil- $\beta$-D-Glucosamidasa), y KIM-1 (Kidney Injury Molecule-1) (Sirota et al., 2011; Bonventre et al., 2010). Esto supone una ventaja a la hora del diagnóstico temprano del daño renal. Centrándonos en el tabaquismo, el doctor Francisco José Sanz Parras demostró en su tesis doctoral que la excreción de KIM-1 es mayor en fumadores que en no fumadores (ambos con los mismos factores de riesgo de daño renal), mientras que los niveles de $\mathrm{CrP}$ permanecen normales en los dos grupos de pacientes (Sanz, 2016).

En este contexto del diagnóstico temprano, en nuestro laboratorio se ha desarrollado un nuevo concepto: la predisposición al daño renal. Así, diferentes fármacos a dosis subtóxicas predisponen a los animales de experimentación al daño renal agudo (DRA), que se desencadena cuando estos animales se exponen a otro fármaco, también a dosis subtóxicas. Así, los marcadores urinarios de predisposición son sustancias que, sin que el riñón haya desarrollado ninguna alteración patológica, indican que hay riesgo de sufrir daño renal, aun por exposición a sustancias a dosis inferiores al límite tolerable.

\section{Hipótesis y objetivos}

Basándonos en estudios previos de nuestro grupo, es posible que el tabaco predisponga a sufrir DRA, que se desencadenaría por exposición de los pacientes fumadores a otras sustancias nefrotóxicas como algunos fármacos (aminoglucósidos, antivíricos, quimioterápicos, etc.), pruebas diagnósticas (medios de contraste) 
o contaminantes ambientales. De ser así, se podría prevenir el daño antes de su aparición mediante la detección de la predisposición con biomarcadores urinarios.

De acuerdo con esta hipótesis, los objetivos de este estudio son:

- Estudiar si los marcadores urinarios de predisposición: Biomarcador 1 (BM1), Biomarcador 2 (BM2) y Biomarcador 3 (BM3) (encriptados por posible patentabilidad), previamente identificados en nuestro laboratorio, son capaces de diferenciar a los individuos fumadores de los no fumadores.

- Estudiar la relación entre estos biomarcadores y el consumo de tabaco.

\section{Materiales y métodos}

Tras la aprobación del protocolo de recogida de muestras y del documento de información y consentimiento informado por el Comité Ético de Investigación Clínica del Complejo Hospitalario de Cáceres (Hospital Nuestra Señora de la Montaña), se tomaron muestras de orina de voluntarios sanos mayores de edad, de la consulta de Atención Primaria del Centro de Salud de Coria, Cáceres. Se excluyeron pacientes con insuficiencia renal previa diagnosticada o que presentasen factores de riesgo de daño renal (diabetes, hipertensión arterial o consumo de fármacos nefrotóxicos). Se obtuvieron 79 muestras de no fumadores (NF) y 56 de fumadores (F). Los datos de creatinina plasmática, empleada para corregir las distintas proteinurias dependiendo de la diuresis de los pacientes, se obtuvieron del historial clínico del paciente en los últimos 6 meses. Por su parte, la creatininuria, relacionada con la funcionalidad renal, se midió empleando un kit comercial basado en la reacción de Jaffé. La medición de cotinina urinaria, metabolito de nicotina que informa del grado de tabaquismo del paciente, fue realizada por el Reference Laboratory de Barcelona. Finalmente, los biomarcadores se midieron empleando la técnica western blot.

La estadística descriptiva se hizo con el programa Excel@, mientras que para la correlación de las variables se empleó el SPSS®.

\section{Resultados}

\subsection{Caracterización de los pacientes}

Se obtuvieron 135 muestras de orina. Tras analizar las características de los pacientes, se observó que ambos grupos, exceptuando el hábito tabáquico, son estadísticamente similares y tienen niveles normales de $\operatorname{CrP}(0,6-1,3 \mathrm{mg} / \mathrm{dL})$ (Medline Plus, 2018) (Tabla 1). 
Tabla 1. Características de los pacientes del estudio. Los valores están expresados como la media \pm desviación estándar de la media. Test chi cuadrado: $\mathrm{p}$-valor sexo: 0,280. Test T de Student: p-valor de Edad: 0,159; p-valor de Peso: 0,329; p-valor de Altura: 0,132; p-valor del IMC: 0,768.

\begin{tabular}{cccccccc} 
Grupo & $\begin{array}{c}\text { N. }{ }^{\circ} \text { de } \\
\text { varones }\end{array}$ & $\begin{array}{c}\text { N. }{ }^{\circ} \text { de } \\
\text { mujeres }\end{array}$ & $\begin{array}{c}\text { CrP } \\
(\mathrm{mg} / \mathrm{dL})\end{array}$ & $\begin{array}{c}\text { Edad } \\
(\text { años })\end{array}$ & Peso (kg) & Altura (cm) & $\begin{array}{c}\text { IMC } \\
\left(\mathbf{k g} / \mathrm{m}^{2}\right)\end{array}$ \\
\hline NF & 37 & 42 & $0,81 \pm 0,01$ & $44 \pm 14$ & $72,81 \pm 15,83$ & $168,11 \pm 8,77$ & $25,60 \pm 4,41$ \\
\hline F & 21 & 35 & $0,79 \pm 0,02$ & $42 \pm 11$ & $68,32 \pm 12,63$ & $164,88 \pm 7,69$ & $25,03 \pm 3,84$ \\
\hline
\end{tabular}

\subsection{BM1}

Solo se detectó BM1 en la muestra de orina de un paciente fumador, por lo que su excreción no parece relacionarse con el consumo de tabaco.

\section{3. $B M 2$}

Se detectó BM2 en 4 muestras de orina de pacientes fumadores y 11 de no fumadores. Al representar la distribución de todos los valores, los dos grupos parecen similares, hecho que se corroboró al no encontrar diferencias estadísticamente significativas. La excreción de BM2, por tanto, no parece depender del tabaquismo (Figura 1).

\section{4. $B M 3$}

Tras determinar las concentraciones de BM3 en las muestras de orina, se representó la dispersión de los datos (Figura 2), donde se observa que los pacientes del grupo $\mathrm{F}$ excretan mayor cantidad del biomarcador que el grupo NF. Esta diferencia se aprecia incluso antes de aplicar la corrección con la creatininuria. Al comparar las medianas de los grupos, se comprobó que estas son estadísticamente diferentes, por lo que la excreción de BM3 parece estar relacionada con el tabaquismo.

De acuerdo con estos resultados, se estudió la relación de los niveles excretados de BM3 con los de cotinina (Figura 3), pero no se obtuvo correlación estadísticamente significativa entre ambos valores. 


\section{FIgURA 1}

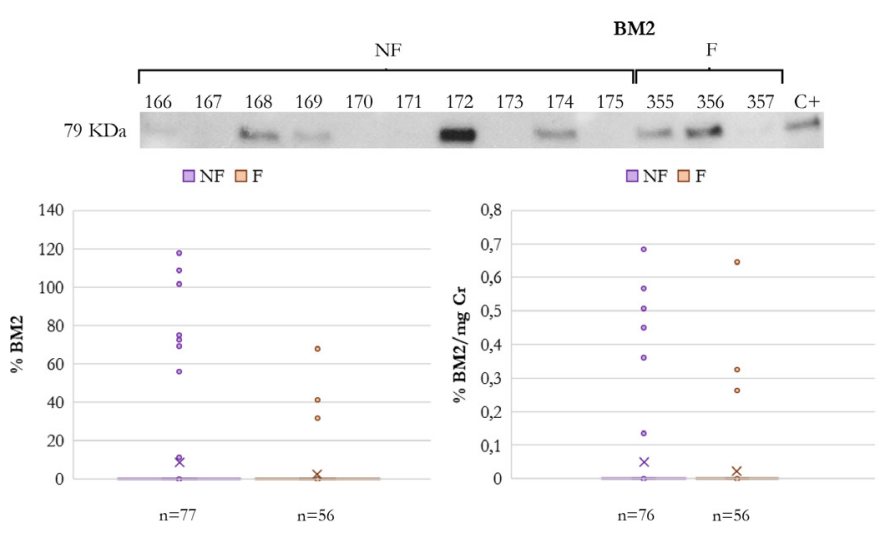

Arriba: WB representativo del análisis de BM2 en las muestras de orina. Los números hacen referencia al código del paciente al que la muestra pertenece. NF: no fumadores; F: fumadores; $\mathrm{C}+$ : control positivo. Abajo: representación en diagrama de box-plot o caja con bigotes de las concentraciones de BM2 medidas en las muestras de orina, incluyendo valores 0. NF: no fumadores; F: fumadores. Prueba U de Mann-Whitney: p-valor de \% BM2: 0,184; p-valor de \% BM2/mg Cr: 0,273.

\section{Figura 2}
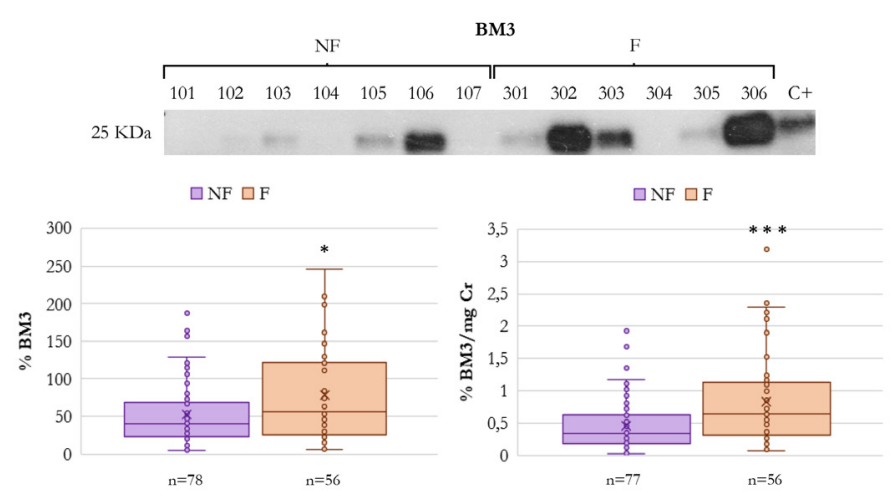

Arriba, WB representativo del análisis de BM3 en las muestras de orina. Los números hacen referencia al código del paciente al que la muestra pertenece. NF: no fumadores; F: fumadores; $\mathrm{C}+$ : control positivo. Abajo, representación en diagrama de box-plot o caja con bigotes de la dispersión de los valores de BM3. Prueba U de Mann-Whitney: p-valor de \% BM3: 0,026; p-valor de \% BM3/mg Cr: 0,0003. *: Diferencia significativa (p-valor $<0,05)$ respecto NF. $*$ : Diferencia significativa $(p$-valor $<0,001)$ respecto $N F$. 
Figura 3. Representación de los valores de BM3 del grupo de fumadores versus los niveles de cotinina. Correlación de Pearson: p-valor de \% BM3: 0,881; p-valor de

$$
\% \mathrm{BM} 3 / \mathrm{mg} \text { Cr: 0,204. }
$$
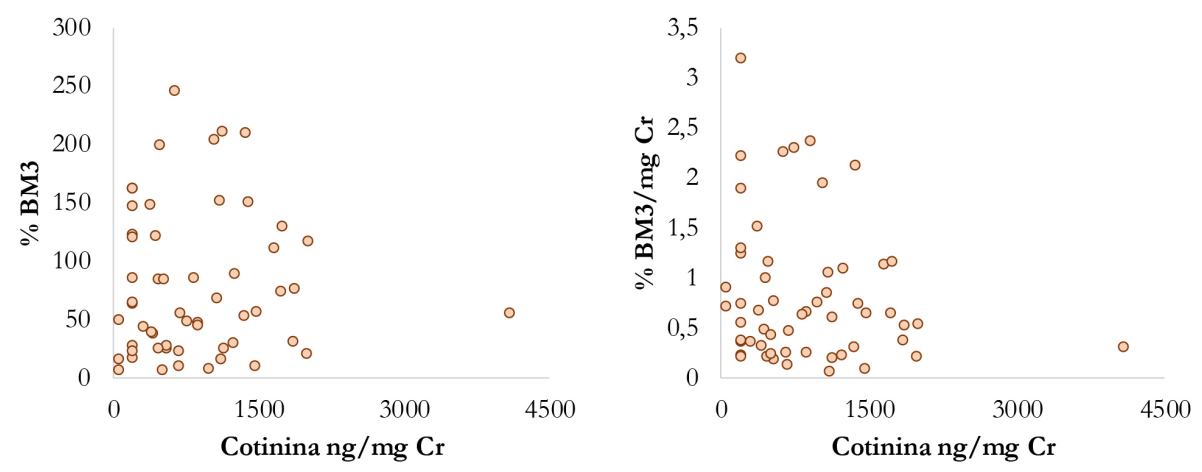

\section{Discusión}

Tras haberse demostrado que el tabaco es un factor de riesgo en el daño renal (Rocco et al., 1996; Orth et al., 2002; Orth et al., 2008) y saber que KIM-1 puede ser un marcador temprano de daño renal asociado al consumo de tabaco (Sanz, 2016), este estudio supone un paso más en el diagnóstico de este riesgo: la identificación de pacientes predispuestos, por su condición de fumadores, a sufrir DRA. A diferencia de los marcadores tempranos, los de predisposición permiten conocer la susceptibilidad sin existir daño renal incipiente. Así, se podría prevenir mejor este riesgo en pacientes fumadores, por ejemplo, evitando o reduciendo la dosis de fármacos o sustancias potencialmente nefrotóxicas. Además, conocer la predisposición podría resultar convincente para dejar de fumar.

De los tres marcadores de predisposición estudiados, solo BM3 mostró diferencias estadísticamente significativas entre los dos grupos, siendo el tabaquismo el único factor distintivo de los estudiados. Al estudiar la correlación entre los niveles de BM3 y cotinina, no parece haber una relación, lo que plantea diversas posibilidades:

- La mayor excreción de BM3 puede ser resultado de la sinergia con otros factores de riesgo ajenos a los criterios de exclusión.

- La predisposición puede estar más relacionada con el tiempo que el paciente lleve como fumador que con la cantidad, factor no contemplado en el estudio. 
M. DE JUAN, M. PRIETO, M. ${ }^{a}$ T. HERNÁNDEZ, F. J. SANZ, A. G. CASANOVA, L. VICENTE, M. PESCADOR, A. I. MORALES

PREDISPOSICIÓN ADQUIRIDA AL DAÑO RENAL ASOCIADA AL CONSUMO DE TABACO

- El aumento de la población de estudio podría dar lugar a una correlación significativa entre ambos parámetros.

A pesar de no encontrarse una relación directa BM3-cotinina, este marcador nos puede permitir conocer a los pacientes predispuestos incluso antes de aplicar la corrección con la creatininuria, lo que resultaría más sencillo en su aplicación clínica.

Finalmente, aún queda por conocer si estos pacientes predispuestos desarrollarán DRA en un futuro como consecuencia de la exposición a algún otro agente nefrotóxico o al mismo hábito tabáquico, es decir, demostrar que el BM3 es un marcador de predisposición a DRA en pacientes fumadores.

En cuanto a BM1 y BM2, no parecen ser marcadores de predisposición relacionados al tabaquismo, por lo que en los pacientes en los que sí se ha detectado es posible que la aparición esté causada por otros agentes diferentes.

\section{Conclusiones}

- Los pacientes fumadores excretan mayor cantidad de BM3 que los no fumadores.

- Este hecho podría indicar predisposición de los fumadores al DRA.

- La cantidad excretada de BM3 no parece tener una correlación directa con el grado de tabaquismo.

- Conocer la predisposición al DRA permitiría prevenir mejor su aparición y concienciar más al paciente fumador sobre los riesgos de su hábito.

\section{Bibliografía}

Bonventre JV, Vaidya VS, Schmouder R, Feig P, Dieterle F. Next-generation biomarkers for detecting kidney toxicity. Nat Biotechnol. 2010; 28(5):436-440.

Christiansen JS. Cigarette smoking and prevalence of microangiopathy in juvenile-onset insulin-dependent diabetes mellitus. Diabetes. 1978; 1(3):146-149.

Esteller Pérez A, Cordero Sánchez M. Fundamentos de Fisiopatología. 1. ${ }^{a}$ ed. Madrid: MacGraw-Hill Interamericana; 1998.

FDA | Cigarettes [Internet]. fda.gov. [citado 4 de enero de 2018; actualizado 16 de agosto de 2019]. Disponible en: https://www.fda.gov/TobaccoProducts/Labeling/ProductsIngredientsComponents/ucm482563.htm.

FDA | Harmful and Potentially Harmful Constituents in Tobacco Products and Tobacco Smoke: Established List [Internet]. fda.gov. [citado 4 de enero de 2018; actualizado 7 de octubre de 2019]. Disponible en: https://www.fda.gov/TobaccoProducts/Labeling/ RulesRegulationsGuidance/ucm297786.htm. 
MedlinePlus enciclopedia médica | Examen de creatinina en la sangre [Internet]. medlineplus.gov. [citado 20 de junio de 2018; actualizado 2 de octubre de 2019]. Disponible en: https://medlineplus.gov/spanish/ency/article/003475.htm.

OMS | Tabaco [Internet]. WHO.int. [citado 22 de septiembre de 2017; actualizado 26 de julio de 2019]. Disponible en: http://www.who.int/mediacentre/factsheets/fs339/es/.

Orth SR, Hallan SI. Smoking: A Risk Factor for Progression of Chronic Kidney Disease and for Cardiovascular Morbidity and Mortality in Renal Patients-Absence of Evidence or Evidence of Absence? Clin J Am Soc Nephrol. 2008; 3(1):226-236.

Orth SR, Ritz E. The renal risks of smoking: an update. Curr Opin Nephrol Hypertens. 2002; 11(5):483-488.

Perazzi B, Angerosa M. Creatinina en sangre: calidad analítica e influencia en la estimación del Îndice de Filtrado Glomerular. Acta Bioquímica Clínica Latinoam. 2011; 45(2):265-272.

Pickering JW, Endre ZH. The Metamorphosis of Acute Renal Failure to Acute Kidney Injury | IntechOpen [Internet]. intechopen.com. [citado 30 de junio de 2018; actualizado 2 de marzo de 2012]. Disponible en: https://www.intechopen.com/books/ basic-nephrology-and-acute-kidney-injury.

Repetto Jiménez M. Toxicología Fundamental. 1. a ed. España: Ediciones Díaz de Santos, S.A.; 2013.

Rocco MV, Soucie JM, Reboussin DM, McClellan WM. Risk factors for hospital utilization in chronic dialysis patients. J Am Soc Nephrol. 1996; 7(6):889-896.

Sanz Parras FJ. Biomarcadores de daño renal temprano asociados al consumo de tabaco. Universidad de Salamanca; 2016.

Sirota JC, Klawitter J, Edelstein CL. Biomarkers of Acute Kidney Injury. J Toxicol. 2011; 2011:1-10. 